mononuclear cells were analysed by flow cytometry. Patients completed a scored questionnaire addressing sun exposure history prior to disease onset. The questionnaire, flow cytometry and ELISA results were analysed using Mann-Whitney test.

Results Questionnaire responses indicate increased sun exposure prior to disease onset in SLE patients with skin disease when compared to SLE patients without skin disease (median score $=60$ versus 32, respectively; $\mathrm{p}<0.05$ ). Anti-desmoglein-3 auto-antibody levels were higher in the serum of SLE patients with skin disease than in patients without skin disease (median $=0.571$ versus $0.123 \mathrm{IU}$, respectively; $\mathrm{p}<0.05$ ). T-follicular helper (TFH) cells stimulate B-cells to produce autoantibodies via IL-21. There was a trend to enhanced IL-21 production in SLE with skin lesions compared to SLE without skin (median $=34$ versus 19\%, respectively).

Conclusions SLE patients with skin disease have a history of higher antecedent sun exposure consistent with the hypothesis that sun exposure is an environmental trigger. The resulting immune activation of the skin may be reflected in aberrant skin-specific antibody production and heightened IL-21 secretion by TFH cells.

\section{RISK FACTORS ASSOCIATED WITH THE OCCURRENCE OF AUTOIMMUNE HEMOLYTIC ANAEMIAANEMIA IN SYSTEMIC LUPUS ERYTHEMATOSUS}

${ }^{1}$ A Kurniawan*, ${ }^{1} \mathrm{NPH}$ Lugito, ${ }^{1} \mathrm{~S}$ Sumantri, ${ }^{2} \mathrm{AS}$ Soetijpto, ${ }^{2} \mathrm{~V}$ Kusnaidi, ${ }^{2} \mathrm{D}$ Hadi, ${ }^{2} \mathrm{LM}$ Rouly. ${ }^{1}$ University of Pelita Harapan, Internal medicine, Tangerang, Indonesia; ${ }^{2}$ University of Pelita Harapan, School of Medicine, Tangerang, Indonesia

\subsection{6/lupus-2017-000215.218}

Background and aims Almost all systemic lupus erythematosus (SLE) patients develop haematological abnormalities during their disease course. Autoimmune hemolytic anaemia (AIHA) was reported in 5\%-14\% of SLE patients which is usually mediated by warm-type IgG anti-erythrocyte antibodies. There is still paucity data about risk factors associated with the occurrence of AIHA in SLE patients. The aim of this study is to know risk factors associated with the occurrence of AIHA in SLE patients.

Methods This study was a retrospective cohort single centre study from 2013-2015 from our general hospital, Karawaci, Tangerang, Banten, Indonesia. The criteria of SLE patients were using American College of Rheumatology (ACR) criteria. The data were from our medical records database. The criteria of AIHA were based on American Society Haematology (ASH) criteria. Clinical data and risk factors of AIHA patients were reviewed and analysed. Anti-nuclear antibody (ANA) and antidsDNA were detected using indirect immunofluorescence test (IFA-Bio-Rad, USA).

Results Fifty-seven patients were included, of whom 93\% were female with a median age of 36 (12-72) year old. AIHA patient found in $57.9 \%$ of the patients with positive IgG antibody to erythrocyte. ANA was positive in $84.2 \%$ and antidsDNA was positive $75.4 \%$. Positive ANA, OR 1.91 (0.45$8.02)$; positive anti-dsDNA 2.25 (0.66-7.76); decreased complement3 (C3) 0.77 (0.23-2.51); decreased C4 $0.67 \quad$ (0.212.16); decreased albumin level 0.82 (0.23-2.92); thrombocytopenia 3.19 (1.01-10.05), leucopenia $0.95(0.30-3.0)$ did not significantly related to AIHA.

Conclusions The proportion of AIHA in SLE patients 57.9\%. Positive ANA, anti-dsDNA, decreased C3, C4, hypoalbuminemia, thrombocytopenia, and leucopenia were not statistically significant.

\section{EFFECT OF COMPLETE OR PARTIAL PROTEINURIA RECOVERY COMPARED TO NO RECOVERY AT 2 YEARS AFTER THE DIAGNOSIS OF LUPUS NEPHRITIS ON LONG TERM OUTCOMES}

1J Medina-Rosas, 'D Gladman*, 2J Su, 'M Urowitz, 'Z Touma. 'University of Toronto, Medicine- Rheumatology, Toronto, Canada; ${ }^{2}$ University Health Network, Rheumatology, Toronto, Canada

\subsection{6/lupus-2017-000215.219}

Background and aims To evaluate the effect of Complete Recovery (CR), Partial Recovery (PR), and No Recovery (NR) at 2 years from diagnosis of $\mathrm{LN}$ on long term outcomes.

Methods Patients with LN (proteinuria in 24 hour urine [24H$\mathrm{P}]>0.5 \mathrm{~g}$ /day) were studied. At 2 years from $\mathrm{LN}$, patients were divided into CR, PR and NR. Long-term outcomes were studied up to 15 years. CR was defined as normal $24 \mathrm{H}-\mathrm{P}$, PR as a reduction $\geq 50 \%$ in $24 \mathrm{H}-\mathrm{P}$ without achieving $\mathrm{CR}$ and $\mathrm{NR}$ as a reduction $<50 \% 24$ hour-P compared to baseline. Long term outcomes: Renal outcomes (low eGFR $<15 \mathrm{~mL}$ / min, end-stage renal disease requiring dialysis or transplantation [ESRD], and a Composite Renal Outcome [low eGFR or ESRD]); Cardio-Vascular (CV) outcomes (angina or myocardial infarction); Damage (SDI $\geq 1)$; and Death. Time-independent and time-dependent Cox proportional hazards models were applied to describe the effect of CR, PR or NR on long-term outcomes.

Results Of 277 patients, 63.9\% achieved CR, 18.41\% PR, and $9.75 \% \mathrm{NR}$ at 2 years. CR protected from all long-term outcomes compared to PR and NR on Kaplan-Meier analysis and Cox model (Figure 1). CR protected against CV outcomes only in the Cox model. Compared to NR, PR only protected against low eGFR. Neither CR nor PR protected against damage. On time-dependent analysis, when comparing CR to NR and PR to NR, only NR was a risk factor for ESRD when compared to $\mathrm{CR}(\mathrm{HR}=3.93)$.

Conclusions CR protects against $\mathrm{CV}$ and renal outcomes, and mortality. PR protects against low eGFR.

\section{RELATIONSHIP BETWEEN INCREASED LEVELS OF ANTI- DSDNA WITH CLINICAL SYMPTOMS IN PATIENTS WITH SLE}

${ }^{1}$ B Marpaung. ${ }^{1}$ Faculty of Medicine- University of Sumatera Utara- Adam Malik General Hospital, Department of Internal Medicine, Medan, Indonesia

\subsection{6/lupus-2017-000215.220}

Background Systemic Lupus Erythematosus (SLE) is an autoimmune rheumatic disease characterised by widespread inflammation and affectsany organ or system in the body. Many autoimmune diseases result in autoantibody production, but anti-dsDNA antibodies are highly specific to SLE. Previous study found that Anti-dsDNA antibodies are associated with severe clinical manifestations of lupus.

Objective To examine the association between anti-dsDNA level with clinical features and laboratory findings in patients with SLE.

Methods This cross-sectional study was conducted in the Haji Adam Malik General Hospital Medan in August-October 2016 


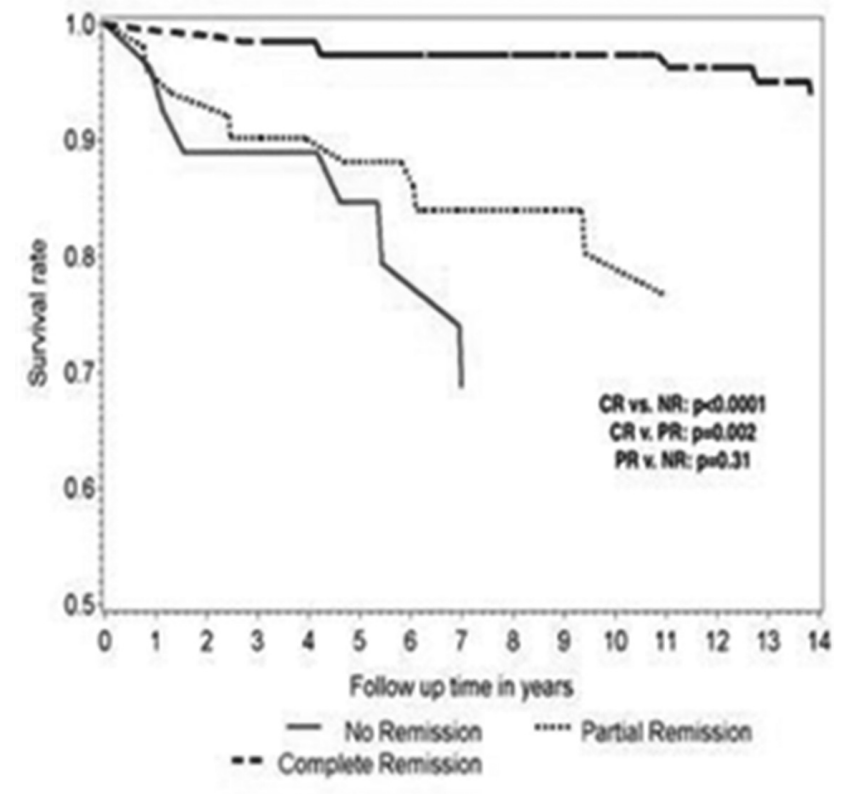

Abstract 219 Figure 1 Kaplan Meier analysis of long term outcome of low eGFR ( $<15 \mathrm{~mL} / \mathrm{min})$ among patients who acheived $C R, P R$ or NR at 2 years of $L N$ diagnosis

from 81 patients with SLE.We examined anti-dsDNA level, clinical features and kidney laboratory profile in all patient. The obtained data were statistically analysed.

Results 81 SLE patients with mean level of anti-dsDNA $294 \mathrm{IU} / \mathrm{ml}$ (6.1-1317). There is no significant relationship between increased level of Anti-dsDNA with other clinical manifestations $(p>0.05)$. There are significant relationships between increased level of Anti-dsDNA with ureum level $(\mathrm{p}=0.016), \quad$ thrombocytopenia $\quad(\mathrm{p}=0.001), \quad$ leucopenia $(\mathrm{p}=0.006)$, kidney disorder $(\mathrm{p}=0.049)$ and urine protein $(\mathrm{p}=0.028)$.Arthritis is the most frequent clinical manifestation (96.3\%) from this study followed by malar rash $(77.8 \%)$ and photo sensitivity (40.7\%).

Conclusions Elevated anti-ds DNA level was not correlated with clinical symptoms but there is significant correlation with haematology disorder and kidney laboratory profiles of SLE patients.

\section{SELECTED NAILFOLD CAPILLAROSCOPY PARAMETERS ARE PREDICTIVE OF SLE ONSET IN CONNECTIVE TISSUE DISEASES SUBGROUP}

${ }^{1}$ M Meroni*, ${ }^{1} \mathrm{M}$ Limonta, ${ }^{2} \mathrm{M}$ Cutolo. ${ }^{1}$ ASST Papa Giovanni XXIII, Rheumatology, Bergamo, Italy; ${ }^{2}$ University of Genova, Genova, Italy

\subsection{6/lupus-2017-000215.221}

Background and aims Nailfold capillaroscopy (NVC) is an useful, non-invasive, reproducible and cost-effective favourable diagnostic tool able to assess the shape of capillaries in the periungal region and the presence of their peculiar abnormalities, essential in the differential diagnosis of connective tissue diseases (CTD).

Methods The aim of the study was to evaluate if selected NVC pictures are linked to SLE onset in a cohort of 42
CTD-affected women presenting Raynaud's phenomenon, observed over 36 months. All of them were examined by this method every 6 months. We considered the following NVC parameters: presence of ectasic capillary loops (diameter $\geq 20 \mu \mathrm{m})$; megacapillaries $(\geq 50 \mu \mathrm{m})$; hemosiderin deposits; capillary number reduction; neo-angiogenesis phenomena; micro-vascular array disorganisation. CTD and SLE diagnoses were posed according to the 2015 ACR/SLICC criteria. Qualitative variables were expressed in frequencies; their association, by non-parametric tests. Quantitative variables were assessed by analysis of co-variance.

Results The presence of hemosiderin deposits, ectasic loops and neo-angiogenic phenomena was strongly associated to the clinical subgroup of patients that later developed SLE (12/42 subjects; $\mathrm{OR}=13.5)$. The variable meandering deposits was the more strongly associated to SLE onset $(\mathrm{OR}=8.32 ; \mathrm{p}<0.0101)$. The independent variables ectasic loops $(\mathrm{OR}=12.16)$ and neoangiogenic phenomena $(\mathrm{OR}=6.60)$ were predictive for the persistence of CTD diagnosis.

Conclusions Nailfold capillaroscopy, summarising, can help in CTD management, since the presence of typical capillaroscopic abnormalities seems to be related to the development of SLE.

\section{ASSESSMENT OF THE RISK OF FLARES IN SYSTEMIC LUPUS ERYTHEMATOSUS}

${ }^{1} \mathrm{M}$ Minodora*, ${ }^{2} \mathrm{~L}$ Mazur-Nicorici, ${ }^{3} \mathrm{~V}$ Sadovici-Bobeica, ${ }^{3} \mathrm{M}$ Garabajiu. ${ }^{1}$ State Medical and Pharmaceutical University - Nicolae Testemitanu, Department of Internal Medicine, Chisinau, Moldova; ${ }^{5}$ State Medical and Pharmaceutical University - Nicolae Testemitanu, Department of Internal Medicine Discipline Cardiology, Chisinau, Moldova; ${ }^{3}$ State Medical and Pharmaceutical University - Nicolae Testemitanu, Department of Internal MedicineDiscipline Internal Medicine, Chisinau, Moldova

\subsection{6/lupus-2017-000215.222}

Background and aims To evaluate the risc of flares in SLE patients

Methods A prospective analytic study of SLE patients (SLICC, 2012 criteria) with a fallowed-up in 5 visits: baseline visit, month 3, month 5, month 9 and month 12 visit. At visits disease activity (SLEDAI, SLAM), SELENA/SLEDAI flare index and laboratory tests were assessed.

Results The study included 102 patients, 94,1\% females, age \pm SD $42.4 \pm 13.3$ (range 20-73) years, disease duration \pm SD 93.9 \pm 77.1 (range 0,1-228) months. During fallow-up, 55 flares were enregistered, including 11 severe, with a SLEDAI increase with 3-17 points. The incidence of flares was 0,53 patient/year, the for severe flares - 0,10 patient/year. To assess the risk of flares, potential risk factors were studied (table 1).

The main risk factors were laboratory findings, while only active pulmonary involvement derived from SLAM and antiphospholipid syndrome, as clinical and laboratory variables, associated with the flares in SLE patients.

Conclusions In our study, the incidence of flare in a 12 moths period was $53,9 \%$, including $10,8 \%$ of severe flares. We have determined that patients with high ERS, low $\mathrm{Hb}$ and lymphocytopenia are at risk for flares and antiphospholipid syndrome and pulmonary involvement were the main clinical risk factors in our cohort. 